\title{
Sarcocystis mihoensis n. sp. from Sheep in Japan
}

\author{
Morihiro SAITO, Yutaka SHIBATA, Masanori KUBO ${ }^{1)}$, and Hiroshi ITAGAKI ${ }^{2)}$ \\ Kumagaya Meat Inspection Center Saitama Prefecture, 179-1 Shimomasuda, Kumagaya, Saitama 360, 'National Institute of Animal \\ Health, Tsukuba, Ibaraki 305, and ${ }^{2}$ Azabu University, Fuchinobe, Sagamihara, Kanagawa 229, Japan
}

(Received 19 August 1996/Accepted 29 October 1996)

ABSTRACT. Sarcocystis mihoensis n. sp., a heteroxenous coccidium was detected from sheep in Japan and dogs were experimentally determined as the definitive host. The cysts were $1,300-2,100 \times 200-300 \mu \mathrm{m}$ in size and had the thick cyst wall which was 10 to $12 \mu \mathrm{m}$ thick and provided with radial striated and finger-like villar protrusions. Two 6-month old dogs fed with the cysts passed sporocysts, 15$16 \times 8-9 \mu \mathrm{m}$ in size, in the feces from day 11 to days 57-60 after ingestion. No domestic cats fed with the same cysts shed sporocysts throughout the experimental period. - KEY wORDs: experimental infection, morphology, Sarcocystis mihoensis n. sp., sheep.

Four Sarcocystis species have been recorded from sheep such as $S$. arieticanis, $S$. gigantea, $S$. medusiformis, and $S$. tenella $[1,2,5,7,12]$. Of these species, the final hosts of $S$. tenella and $S$. arieticanis are the dog and those of $S$. gigantea and $S$. medusiformis are the cat [2-9, 13, 15-17]. We detected a new Sarcocystis species of which the cyst wall was morphologically different from that of the species reported from sheep.

The morphology and experimental final host of the present species are described in this paper.

\section{MATERIALS AND METHODS}

Materials: Sixteen Corriedale sheep, 2 to 3 years old, which were raised at a farm in Miho Village, Ibaraki Prefecture were slaughtered at an abattoir in Saitama Prefecture and striated muscle in different parts of body was examined for Sarcocystis cysts.

Methods: Twenty fresh cysts were measured with a micrometer under a light microscope. A part of the cysts were fixed with $10 \%$ formalin and postfixed with $1 \%$ osmic acid. The fixed specimens were then dehydrated in a series of ethanol and dried at the critical point. After platimum was deposited on the specimes, they were observed for the villar protrusions on cyst wall under a scanning electron microscope (Nihon Denshi, JSM-35C). A piece of striated muscle infected with cysts was dissected out and fixed in $10 \%$ formalin, embedded in paraffin and sectioned. Sections were stained with hematoxylin and eosin for histopathological examination. A part of the specimens fixed with formalin and post-fixed with $1 \%$ osmic acid were dehydrated in a series of ethanol and embedded in epoxy resin. Ultrathin sections were stained with uranyl acetate and lead citrate solutions and observed with a transmission electron microscope (Nihon Denshi, $100 \mathrm{CX}$ ).

Two female 6 months-old mongrel dogs and 2 female domestic cats, 6 and 12 months old respectively, were fed with 15 fresh cysts together with a small amount of food, while a female mongrel dog and a cat, 6 months old each, were used as control.
All the inoculated and control animals were daily examined for sporocysts by the sedimentation method using the total amount of feces, with saturated $\mathrm{NaCl}$ solution. Fifty sporocysts just after passed in the feces were measured with a micrometer under a light microscope.

\section{RESULTS}

In 2 of 16 sheep examined, cysts were detected in the diaphragm,masseter, and muscles in the neck, dorsal region, brachium and thigh, especially in the diaphragm in great numbers.

Fresh cysts measured 1,300-2,100 $\times 200-300 \mu \mathrm{m}$ in size. The cyst wall was thick, 10-12 $\mu \mathrm{m}$ in thickness, and of a palisade-like structure. Clear compartments were observed inside the cyst. Histopathological examination revealed that the cyst wall was thick and the villar protrusions were radially arranged. A great number of bradyzoites were observed in the cyst together with a small number of metrocytes which were situated along the cyst wall (Figs. 1 and 2).

Although light microscopy showed that the cyst wall was thick and of "palisade-like" structure, scanning electron micrographs showed that villar protrusions on the cyst wall were finger-like and $8-10 \times 2-2.5 \mu \mathrm{m}$ in size, and were attenuated gradually from the base to about two thirds of length and then abruptly from two thirds to the tip. There were many folds on the surface of villar protrusion from the base to about two thirds of length (Figs. 3 and 4). This structure of villar protrusion was also shown by transmission electron microscopy, but the transverse section of protrusion was occasionally mushroom-like when cut at some angle. Microtubules were observed in the villar protrusion (Figs. 5-7).

All the dogs orally inoculated with the muscle infected with cysts began to pass sporulated sporocysts in the feces on day 11 after inoculation. Sporocysts measured 15-16 $\times$ $8-9 \mu \mathrm{m}$ in size. Patent period was 57 to 60 days. None of the control animals shed sporocysts in the feces (Fig. 8). 


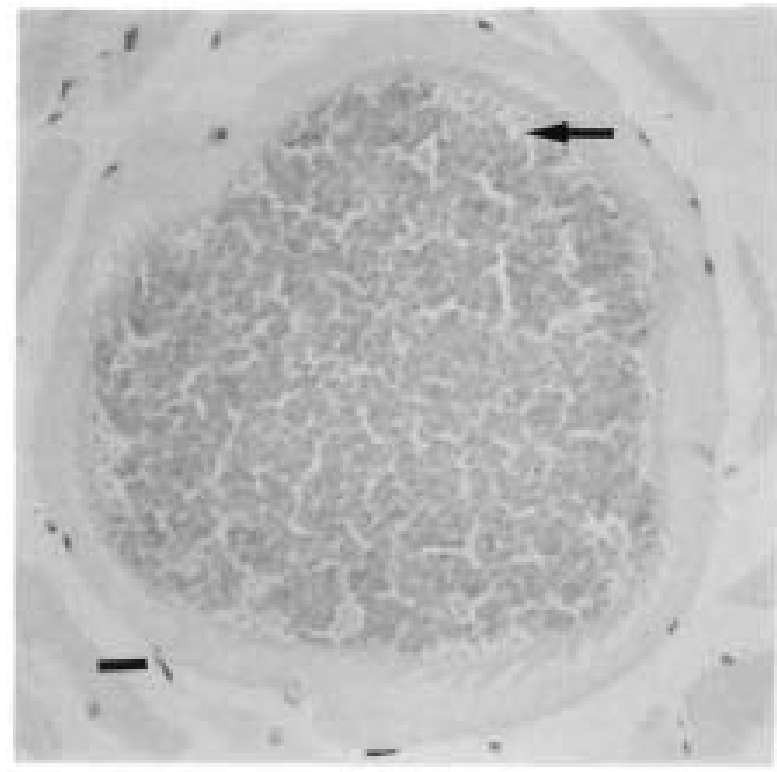

Fig. 1. Cross section of a thick-walled cyst of Sarcocystis mihoensis n. sp. in striated muscle of a sheep. Arrow shows metrocytes. Bar $=10 \mu \mathrm{m}$. H E stain, $\times 400$.

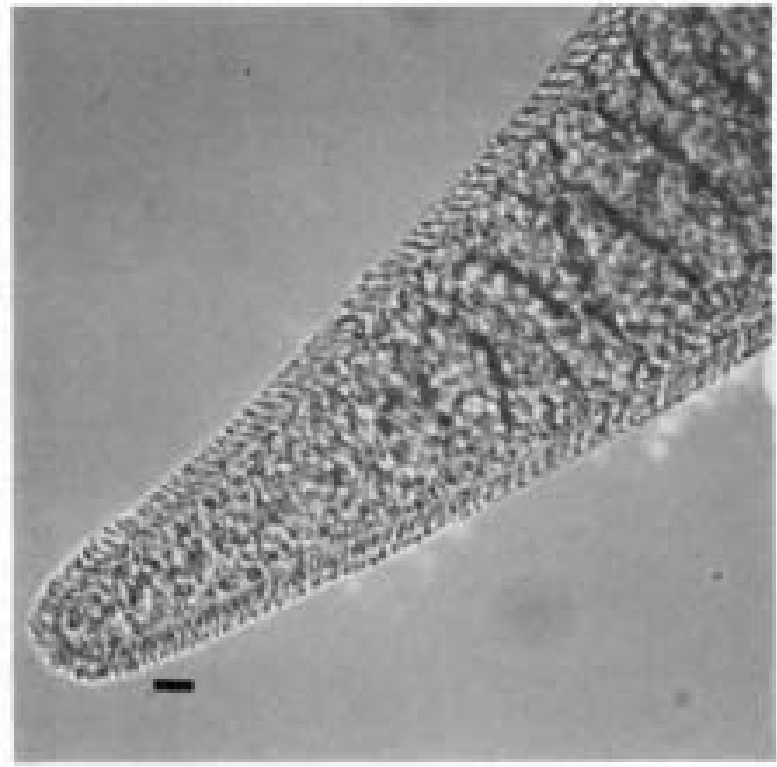

Fig. 2. A fresh cyst removed from striated muscle of a sheep. Bar $=10 \mu \mathrm{m} . \times 400$.

\section{DISCUSSION}

Of the 4 species of Sarcocystis that have been reported from sheep, S. tenella and $S$. arieticanis utilize the dog as the final host and $S$. gigantea and $S$. medusiformis do the cat. The species with the sheep-canid life cycle have far smaller cysts than the sheep-felid species. The cyst of the present species was also smaller than those of the sheepfelid species, but it was the largest among the known

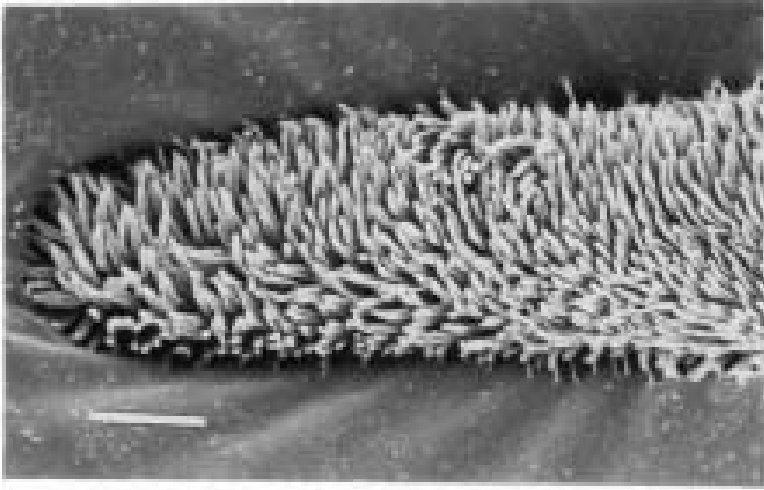

Fig. 3. Scanning electron micrograph (SEM) of a cyst. Bar $=10 \mu \mathrm{m} . \times 1,000$

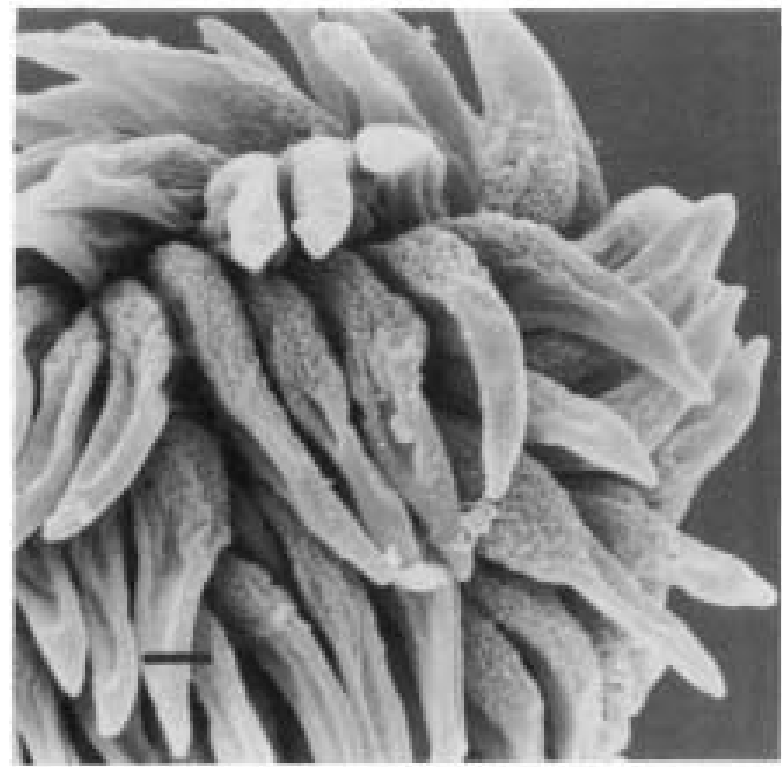

Fig. 4. SEM of cyst, showing finger-like villar protrusions on cyst wall. Bar $=1 \mu \mathrm{m}$.

sheep-canid species (Table 1) [2-9, 13, 16, 17].

The cyst wall of $S$. tenella is observed thick by light microscopy but thin in the other 3 species, $S$. arieticanis, $S$. gigantea and $S$. medusiformis. The present species had the far thicker cyst wall than all the above 4 species, so by this feature, the present species can be distinguished from all the other known species (Table 1) [2, 3, 5, 9-11, 16, 17].

The ultrastructures of villar protrusions detected by scanning and transmission electron microscopy were more useful in distinguishing the present species from the other species. The villar protrusion was finger-like in the present species, whereas it is palisade-like, hair-like, cauliflowerlike, and snake-like in S. tenella, S. arieticanis, S. gigantea and $S$. medusiformis respectively. The presence of microtubule in the villar protrusion also afforded a clue to the identification. The microtubule was found not only in the present species but also in $S$. gigantea and $S$. medusiformis, whereas $S$. tenella and $S$. arieticanis have no 


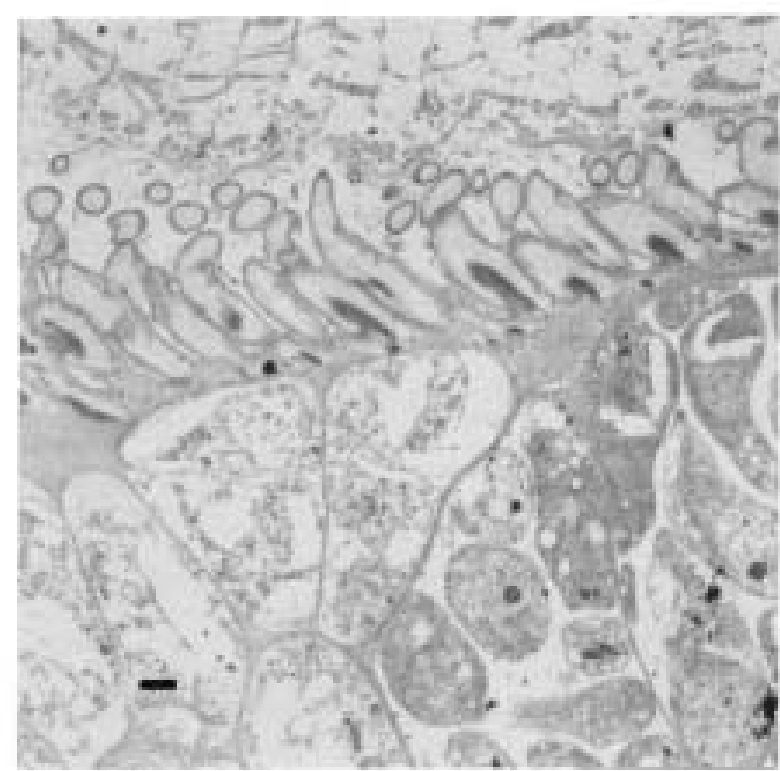

Fig. 5. Transmission electron microcograph (TEM) of a cyst in striated muscle of sheep, showing finger-like villar protrusions and many bradyzoites on and inside of a cyst. Bar $=1 \mu \mathrm{m} . \times 2,000$.

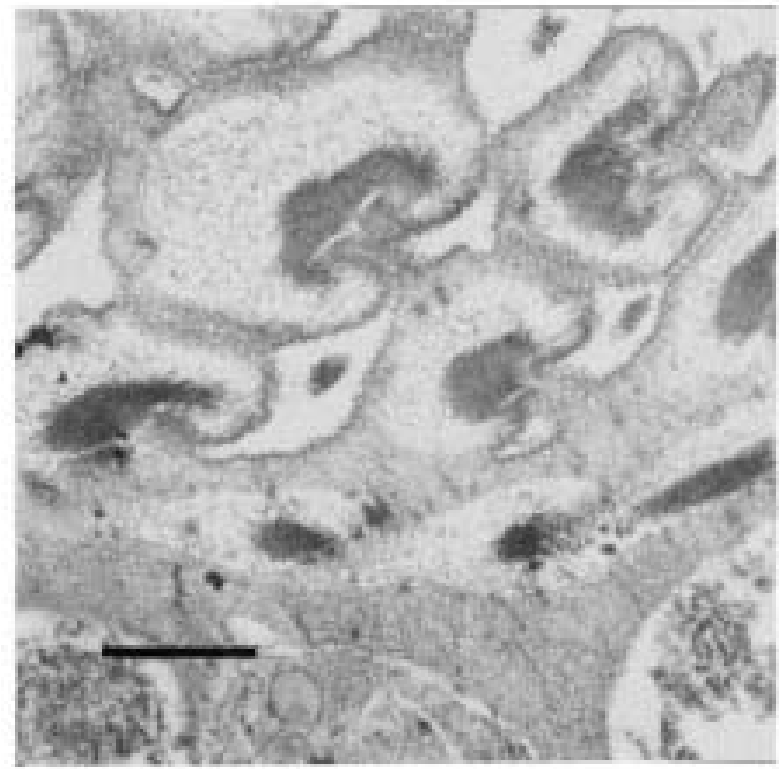

Fig. 6. TEM of a cross section through the finger-like villar protrusions. Note mushroom-like sections of some protrusions. Bar $=1 \mu \mathrm{m} . \times 10,000$.

microtubule (Table 1) [2-5, 9-11, 16, 17].

The prepatent period was not markedly different among the species parasitic in sheep although in S. tenella the period is shorter than those of the other species. Sporocysts are larger in the species with the sheep-canid life cycle than those in the sheep-felid species, but any difference was hardly found in the size among the sheep-canid species [2,

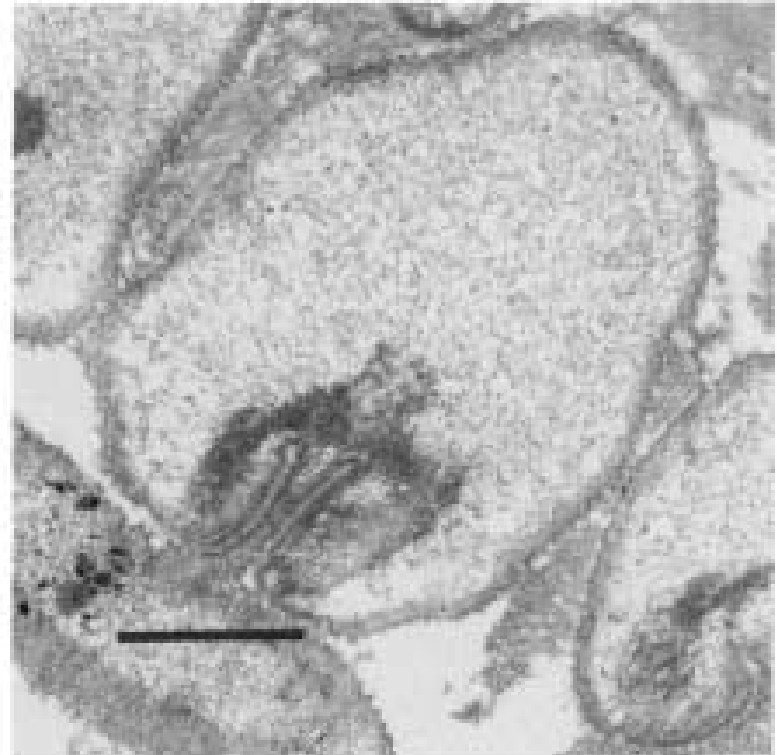

Fig. 7. TEM of cross sections of villar protrusions. Bar $=1 \mu \mathrm{m}$ $\times 12,000$.

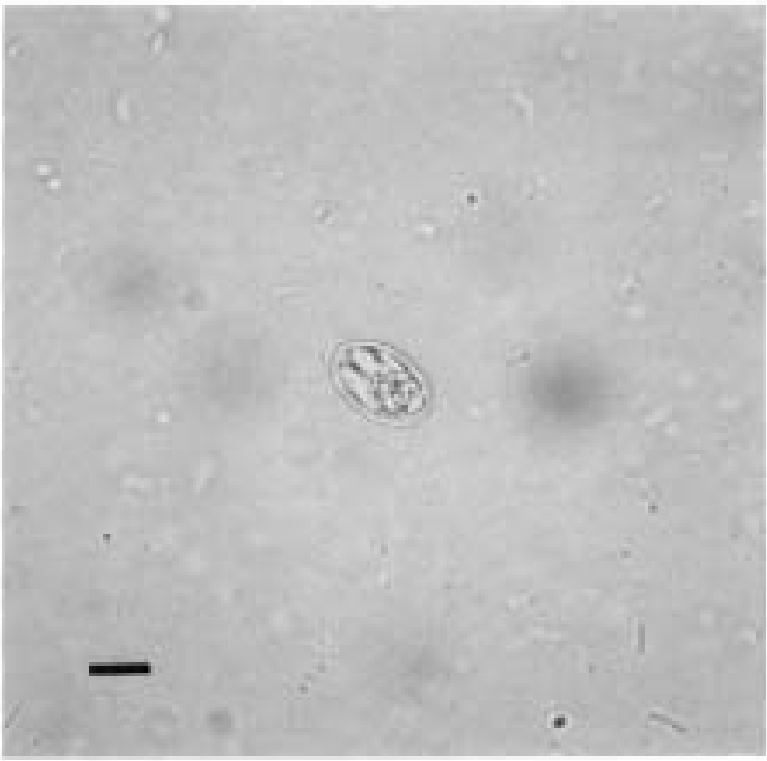

Fig. 8. A fresh sporocyst passed in the feces of dog. Bar=10 $\mu \mathrm{m} . \times 400$.

\section{4, 5, 7, 8, 16].}

From the above characteristics of the morphology of cyst wall, especially of villar protrusion, and of the life cycle, the present species could be discriminated from all the known Sarcocystis species parasitic in sheep, so that the present species was considered to be new to science and was designated as Sarcocystis mihoensis n. sp. 
Table 1. Comparison of biological characteristics of Sarcocystis species parasitic in sheep

\begin{tabular}{|c|c|c|c|c|c|c|c|c|}
\hline \multirow{2}{*}{ Species } & \multirow{2}{*}{$\begin{array}{r}\text { Cyst } \\
\text { length } \\
(\mu \mathrm{m})\end{array}$} & \multirow{2}{*}{$\begin{array}{c}\text { Cyst wall } \\
\text { thickness } \\
(\mu \mathrm{m})\end{array}$} & \multicolumn{2}{|c|}{ Villar protrusion } & \multirow{2}{*}{ Microtubule } & \multirow{2}{*}{ Final host } & \multirow{2}{*}{$\begin{array}{c}\text { Prepatent } \\
\text { period } \\
\text { (in days) }\end{array}$} & \multirow{2}{*}{$\begin{array}{c}\text { Sporocyst } \\
(\mu \mathrm{m})\end{array}$} \\
\hline & & & Shape & Size $(\mu \mathrm{m})$ & & & & \\
\hline S. tenella & $\leqq 700$ & $1-3$ & palisade-like & $3.5 \times 0.5$ & - & $\operatorname{dog}$ & $8-9$ & $14-15 \times 8-10.5$ \\
\hline S. arieticanis & $\leqq 900$ & $<1$ & hair-like & $5-10$ & - & $\operatorname{dog}$ & $\geqq 12$ & $15-16 \times 9-10$ \\
\hline S. gigantea & $\leqq 10000$ & $<2$ & $\begin{array}{c}\text { cauliflower- } \\
\text { like }\end{array}$ & - & + & cat & $10-12$ & $10.5-14.0 \times 8.0-9.7$ \\
\hline S. medusiformis & $\leqq 8000$ & $<2$ & snake-like & - & + & cat & $12-21$ & $10.3-13.0 \times 7.3-8.8$ \\
\hline S. mihoensis n. sp. & $\begin{array}{c}1300- \\
2100\end{array}$ & $10-12$ & finger-like & $\begin{array}{l}8-10 \times \\
2.2-2.5\end{array}$ & + & $\operatorname{dog}$ & 11 & $15-16 \times 8-9$ \\
\hline
\end{tabular}

ACKNOWLEDGMENT. The authors express their gratitude to Dr. Takayuki Kobayashi of Animal Clinic Kobayashi for the materials.

\section{REFERENCES}

1. Ashford, R. W. 1977. The fox, vulpes vulpes, as a final host for Sarcocystis of sheep. Ann. Trop. Med. Parasitol. 71: 2934.

2. Collins, G. H., Atkinson, E. M., and Charleston, W. A. G. 1979. Studies on Sarcocystis species. 3. The macrocystic species of sheep. N. Z. Vet. J. 27: 204-206.

3. Dubey, J. P., Lindsay, D. S., Speer, C. A., Fayer, R., and Livingston, C. W. Jr. 1988. Sarcocystis arieticanis and other Sarcocystis species in sheep in the United States. J. Parasitol. 74: 1033-1038.

4. Dubey, J. P., Speer, C. A., Callis, G., and Blixt, J. A. 1982. Development of sheep-canid cycle of Sarcocystis tenella. Can. J. Zool. 60: 2464-2477.

5. Dubey, J. P., Speer, C. A., and Fayer, R. 1989. pp. 113-120, 146-147, Sarcocystosis of Animals and Man. CRC Press, Boca Raton, Florida.

6. Erber, M. 1982. Life cycle of Sarcocystis tenella in sheep and dog. Z. Parasitenkd. 68: 171-180.

7. Heydorn, A. O. 1985. Zur Entwicklung von Sarcocystis arieticanis n. sp. Berl. Muench. Tieraerztl. Wochenschr. 98: 231-241.

8. Heydorn, A. O., Gestrich, R., Mehlhorn, H., and Rommel, M. 1975. Proposal for a new nomenclature of the Sarcosporidia. Z. Parasitenkd. 48: 73-82.
9. Heydorn, A. O. and Mehlhorn, H. 1987. Fine structure of Sarcocystis arieticanis Heydorn, 1985 in its intermediate and final host (sheep and dog). Zentralbl. Bakteriol. Parasitenkd. Infektionskr. Hyg., I Abt. Orig., A 264: 353-362.

10. Mehlhorn, H., Heydorn, A. O., and Gestrich, R. 1975. Lichtund elektronenmikroskopische Untersuchungen an Cysten von Sarcocystis ovicanis (Heydorn et al. 1975) in der Muskulatur von Schafen. Z. Parasitenkd. 48: 83-93.

11. Mehlhorn, H., Hartley, W. J., and Heydorn, A. O. 1976. A comparative ultrastructural study of the cyst wall of 13 Sarcocystis species. Protistologica 12: 451-467.

12. Moulé, L. T. 1886. Psorospermies du tissu musculaire du mouton. Bull. Mem. Soc. Centr. Med. Vet. 40: 125-129.

13. Obendorf, P. L. and Munday, B. L. 1987. Experimental infection with Sarcocystis medusiformis in sheep. Vet. Parasitol. 24: 59-65.

14. Pomroy, W. E. and Charleston, W. A. G. 1987. Prevalence of dog-derived Sarcocystis spp. in some New Zealand lambs. $N$. Z. Vet. J. 35: 141-142.

15. Saito, M., Hachisu, K., Iwasaki, K., Nakajima, T., Watanabe, A., Moriya, H., and Itagaki, H. 1984. A new simple method for detection of bovine Sarcocystis cysts. J. Jpn. Vet. Med. Assoc. 37: 158-162 (in Japanese with English summary).

16. Saito, M., Shibata, Y., and Itagaki, H. 1996. Sarcocystis arieticanis of sheep in Japan. Jpn. J. Parasitol. 45: 290-294.

17. Saito, M., Shibata, Y., Kobayashi, T., Kobayashi, M., Kubo, M., and Itagaki, H. 1996. Ultrastructure of the cyst wall of Sarcocystis species with canine final host in Japan. J. Vet. Med. Sci. 58: 861-867. 